The Journal of $\mathbf{N}_{\text {onlinear }} \mathbf{S}$ ciences and Applications http://www.tjnsa.com

\title{
APPROXIMATELY PARTIAL TERNARY QUADRATIC DERIVATIONS ON BANACH TERNARY ALGEBRAS
}

\author{
A. JAVADIAN ${ }^{1}$, M. ESHAGHI GORDJI ${ }^{2, *}$ AND M. BAVAND SAVADKOUHI ${ }^{3}$
}

Dedicated to Themistocles M. Rassias on the occasion of his sixtieth birthday

Abstract. Let $A_{1}, A_{2}, \ldots, A_{n}$ be normed ternary algebras over the complex field $\mathbb{C}$ and let $B$ be a Banach ternary algebra over $\mathbb{C}$. A mapping $\delta_{k}$ from $A_{1} \times \cdots \times A_{n}$ into $B$ is called a $k$-th partial ternary quadratic derivation if there exists a mapping $g_{k}: A_{k} \rightarrow B$ such that

$$
\begin{aligned}
\delta_{k}\left(x_{1}, \cdots,\left[a_{k} b_{k} c_{k}\right], \cdots, x_{n}\right) & =\left[g_{k}\left(a_{k}\right) g_{k}\left(b_{k}\right) \delta_{k}\left(x_{1}, \cdots, c_{k}, \cdots, x_{n}\right)\right] \\
& +\left[g_{k}\left(a_{k}\right) \delta_{k}\left(x_{1}, \cdots, b_{k}, \cdots, x_{n}\right) g_{k}\left(c_{k}\right)\right] \\
& +\left[\delta_{k}\left(x_{1}, \cdots, a_{k}, \cdots, x_{n}\right) g_{k}\left(b_{k}\right) g_{k}\left(c_{k}\right)\right]
\end{aligned}
$$

and

$$
\begin{array}{r}
\delta_{k}\left(x_{1}, \cdots, a_{k}+b_{k}, \cdots, x_{n}\right)+\delta_{k}\left(x_{1}, \cdots, a_{k}-b_{k}, \cdots, x_{n}\right) \\
=2 \delta_{k}\left(x_{1}, \cdots, a_{k}, \cdots, x_{n}\right)+2 \delta_{k}\left(x_{1}, \cdots, b_{k}, \cdots, x_{n}\right)
\end{array}
$$

for all $a_{k}, b_{k}, c_{k} \in A_{k}$ and all $x_{i} \in A_{i}(i \neq k)$. We prove the Hyers-UlamRassias stability of the partial ternary quadratic derivations in Banach ternary algebras.

\section{INTRODUCTION}

The stability of functional equations was first introduced by Ulam [41] in 1940 . More precisely, he proposed the following problem: given a group $G_{1}$, a metric group $\left(G_{2}, d\right)$, and a positive number $\epsilon$, does there exists a $\delta>0$ such that if a function $f: G_{1} \rightarrow G_{2}$ satisfies the inequality $d(f(x y), f(x) f(y))<\delta$

Date: Received: September 28, 2010; Revised: November 22, 2010.

(c) 2010 N.A.G

*Corresponding author.

2000 Mathematics Subject Classification. Primary 46K05, 39B82, 39B52, 47B47.

Key words and phrases. Hyers-Ulam-Rassias stability; Banach ternary algebra; Partial ternary quadratic derivation. 
for all $x, y \in G_{1}$, then there exists a homomorphism $T: G_{1} \rightarrow G_{2}$ such that $d(f(x), T(x))<\epsilon$ for all $x \in G_{1}$ ? As mentioned above, when this problem has a solution, we say that the homomorphisms from $G_{1}$ to $G_{2}$ are stable. In 1941, Hyers [31] gave a partial solution of Ulam's problem for the case of approximate additive mappings under the assumption that $G_{1}$ and $G_{2}$ are Banach spaces. In 1978, Rassias [39] generalized the theorem of Hyers by considering the stability problem with unbounded Cauchy differences. This phenomenon of stability that was introduced by Rassias [39] is called the Hyers-Ulam-Rassias stability. The following is according to Rassias' theorem.

Theorem 1.1. Let $f: E \rightarrow E^{\prime}$ be a mapping from a norm vector space $E$ into a Banach space $E^{\prime}$ subject to the inequality

$$
\|f(x+y)-f(x)-f(y)\| \leq \epsilon\left(\|x\|^{p}+\|y\|^{p}\right)
$$

for all $x, y \in E$, where $\epsilon$ and $p$ are constants with $\epsilon>0$ and $p<1$. Then there exists a unique additive mapping $T: E \rightarrow E^{\prime}$ such that for all $x \in E$,

$$
\|f(x)-T(x)\| \leq \frac{2 \epsilon}{2-2^{p}}\|x\|^{p} .
$$

This new concept is known as Hyers-Ulam-Rassias stability of functional equations (see [27], 28, [30, 32, 33], 36] and [38]). The functional equation

$$
f(x+y)+f(x-y)=2 f(x)+2 f(y)
$$

is related to symmetric bi-additive function. It is natural that this equation is called a quadratic functional equation. In particular, every solution of the quadratic equation (1.1) is said to be a quadratic function. It is well known that a function $f$ between real vector spaces is quadratic if and only if there exists a unique symmetric bi-additive function $B$ such that $f(x)=B(x, x)$ for all $x$ (see [34]). The bi-additive function $B$ is given by

$$
B(x, y)=\frac{1}{4}(f(x+y)-f(x-y))
$$

Hyers-Ulam-Rassias stability problem for the quadratic functional equation (1.1) was proved by Skof for functions $f: A \longrightarrow B$, where $\mathrm{A}$ is normed space and B Banach space (see [4], [6], [29] and [40]). For more detailed definitions of such terminologies, we can refer to [1], [7]-[14], [16], [19]-[22] and [24].

A ternary (associative) algebra $(A,[])$ is a linear space $A$ over a scalar field $\mathbb{F}=\mathbb{R}$ or $\mathbb{C}$ equipped with a linear mapping, the so-called ternary product, [ ] : $A \times A \times A \rightarrow A$ such that $[[a b c] d e]=[a[b c d] e]=[a b[c d e]]$ for all $a, b, c, d, e \in A$. This notion is a natural generalization of the binary case. Indeed if $(A, \odot)$ is a usual (binary) algebra then $[a b c]:=(a \odot b) \odot c$ induced a ternary product making $A$ in to a ternary algebra which will be called trivial. By a Banach ternary algebra we mean a ternary algebra equipped with a complete norm $\|$.$\| such that$ $\|[a b c]\| \leq\|a\|\|b\|\|c\|$ for all $a, b, c \in A$. It seems that approximate derivations were first investigated by Jun and Park [33]. Recently, the stability of derivations has been investigated by some authors; see [2, 5, 23, 26] and references therein. For more detailed definitions of such terminologies, we can refer to [3], 15], [17], [18, ,25] 
and [37].

\section{Main results}

Let $A_{1}, A_{2}, \ldots, A_{n}$ be normed ternary algebras over the complex field $\mathbb{C}$ and let $B$ be a Banach ternary algebra over $\mathbb{C}$. A mapping $\delta_{k}$ from $A_{1} \times \cdots \times A_{n}$ into $B$ is called a $k$-th partial ternary quadratic derivation if there exists a mapping $g_{k}: A_{k} \rightarrow B$ such that

$$
\begin{aligned}
\delta_{k}\left(x_{1}, \cdots,\left[a_{k} b_{k} c_{k}\right], \cdots, x_{n}\right) & =\left[g_{k}\left(a_{k}\right) g_{k}\left(b_{k}\right) \delta_{k}\left(x_{1}, \cdots, c_{k}, \cdots, x_{n}\right)\right] \\
& +\left[g_{k}\left(a_{k}\right) \delta_{k}\left(x_{1}, \cdots, b_{k}, \cdots, x_{n}\right) g_{k}\left(c_{k}\right)\right] \\
& +\left[\delta_{k}\left(x_{1}, \cdots, a_{k}, \cdots, x_{n}\right) g_{k}\left(b_{k}\right) g_{k}\left(c_{k}\right)\right]
\end{aligned}
$$

and

$$
\begin{array}{r}
\delta_{k}\left(x_{1}, \cdots, a_{k}+b_{k}, \cdots, x_{n}\right)+\delta_{k}\left(x_{1}, \cdots, a_{k}-b_{k}, \cdots, x_{n}\right) \\
=2 \delta_{k}\left(x_{1}, \cdots, a_{k}, \cdots, x_{n}\right)+2 \delta_{k}\left(x_{1}, \cdots, b_{k}, \cdots, x_{n}\right)
\end{array}
$$

for all $a_{k}, b_{k}, c_{k} \in A_{k}$ and all $x_{i} \in A_{i}(i \neq k)$. We denote that $0_{k}, 0_{B}$ are zero elements of $A_{k}, B$, respectively.

Theorem 2.1. Let $p \geq 0$ be given with $p<2$ and let $\theta$ be nonnegative real numbers. Let $F_{k}: A_{1} \times \cdots \times A_{n} \rightarrow B$ be a mapping with $F_{k}\left(x_{1}, \cdots, 0_{k}, \cdots, x_{n}\right)=$ $0_{B}$. Suppose that there exists a quadratic mapping $g_{k}: A_{k} \rightarrow B$ such that

$$
\begin{gathered}
\| F_{k}\left(x_{1}, \cdots, a_{k}+b_{k}, \cdots, x_{n}\right)+F_{k}\left(x_{1}, \cdots, a_{k}-b_{k}, \cdots, x_{n}\right) \\
-2 F_{k}\left(x_{1}, \cdots, a_{k}, \cdots, x_{n}\right)-2 F_{k}\left(x_{1}, \cdots, b_{k}, \cdots, x_{n}\right) \| \\
\leq \theta\left(\left\|a_{k}\right\|^{p}+\left\|b_{k}\right\|^{p}\right) \\
\| F_{k}\left(x_{1}, \cdots,\left[a_{k} b_{k} c_{k}\right], \cdots, x_{n}\right)-\left[g_{k}\left(a_{k}\right) g_{k}\left(b_{k}\right) F_{k}\left(x_{1}, \cdots, c_{k}, \cdots, x_{n}\right)\right] \\
-\left[g_{k}\left(a_{k}\right) F_{k}\left(x_{1}, \cdots, b_{k}, \cdots, x_{n}\right) g_{k}\left(c_{k}\right)\right]-\left[F_{k}\left(x_{1}, \cdots, a_{k}, \cdots, x_{n}\right) g_{k}\left(b_{k}\right) g_{k}\left(c_{k}\right)\right] \| \\
\leq \theta\left(\left\|a_{k}\right\|^{p}+\left\|b_{k}\right\|^{p}+\left\|c_{k}\right\|^{p}\right)
\end{gathered}
$$

for all $a_{k}, b_{k}, c_{k} \in A_{k}, x_{i} \in A_{i}(i \neq k)$. Then there exists a unique $k$-th partial ternary quadratic derivation $\delta_{k}: A_{1} \times \cdots \times A_{n} \rightarrow B$ such that

$$
\left\|F_{k}\left(x_{1}, \cdots, x_{n}\right)-\delta_{k}\left(x_{1}, \cdots, x_{n}\right)\right\| \leq \frac{2 \theta}{4-2^{p}}\left\|x_{k}\right\|^{p}
$$

holds for all $x_{i} \in A_{i}(i=1,2, \cdots, n)$.

Proof. In (2.1), putting $a_{k}=b_{k}=x_{k}$, we have

$$
\left\|F_{k}\left(x_{1}, \cdots, 2 x_{k}, \cdots, x_{n}\right)-4 F_{k}\left(x_{1}, \cdots, x_{k}, \cdots, x_{n}\right)\right\| \leq 2 \theta\left\|x_{k}\right\|^{p},
$$

that is,

$$
\left\|F_{k}\left(x_{1}, \cdots, x_{k}, \cdots, x_{n}\right)-\frac{1}{4} F_{k}\left(x_{1}, \cdots, 2 x_{k}, \cdots, x_{n}\right)\right\| \leq \frac{\theta}{2}\left\|x_{k}\right\|^{p}
$$


for all $x_{i} \in A_{i}(i=1,2, \cdots, n)$. One can use induction on $m$ to show that

$$
\begin{aligned}
& \left\|F_{k}\left(x_{1}, \cdots, x_{k}, \cdots, x_{n}\right)-\frac{1}{2^{2 m}} F_{k}\left(x_{1}, \cdots, 2^{m} x_{k}, \cdots, x_{n}\right)\right\| \\
& \quad \leq \frac{\theta}{2} \sum_{i=0}^{m-1} 2^{i(p-2)}\left\|x_{k}\right\|^{p}
\end{aligned}
$$

for all $x_{i} \in A_{i}(i=1,2, \cdots, n)$ and all non-negative integers $m$. Hence

$$
\begin{gathered}
\left\|\frac{1}{2^{2 j}} F_{k}\left(x_{1}, \cdots, 2^{j} x_{k}, \cdots, x_{n}\right)-\frac{1}{2^{2(m+j)}} F_{k}\left(x_{1}, \cdots, 2^{(m+j)} x_{k}, \cdots, x_{n}\right)\right\| \\
\leq \frac{\theta}{2} \sum_{i=j}^{m+j-1} 2^{i(p-2)}\left\|x_{k}\right\|^{p}
\end{gathered}
$$

for all non-negative integers $m$ and $j$ with $m \geq j$ and all $x_{i} \in A_{i}(i=1,2, \cdots, n)$. It follows from $p<2$ that the sequence $\left\{\frac{1}{2^{2 m}} F_{k}\left(x_{1}, \cdots, 2^{m} x_{k}, \cdots, x_{n}\right)\right\}$ is Cauchy. Due to the completeness of $B$, this sequence is convergent. So one can define the mapping $\delta_{k}: A_{1} \times \cdots \times A_{n} \rightarrow B$ given by

$$
\delta_{k}\left(x_{1}, \cdots, x_{n}\right)=\lim _{m \rightarrow \infty} \frac{1}{2^{2 m}} F_{k}\left(x_{1}, \cdots, 2^{m} x_{k}, \cdots, x_{n}\right)
$$

for all $x_{i} \in A_{i}(i=1, \cdots, n)$. In $(2.1)$, replacing $a_{k}, b_{k}$ with $2^{m} a_{k}, 2^{m} b_{k}$, respectively, we obtain that

$$
\begin{array}{r}
\| \frac{1}{2^{2 m}} F_{k}\left(x_{1}, \cdots, 2^{m}\left(a_{k}+b_{k}\right), \cdots, x_{n}\right)+\frac{1}{2^{2 m}} F_{k}\left(x_{1}, \cdots, 2^{m}\left(a_{k}-b_{k}\right), \cdots, x_{n}\right) \\
-\frac{2}{2^{2 m}} F_{k}\left(x_{1}, \cdots, 2^{m} a_{k}, \cdots, x_{n}\right)-\frac{2}{2^{2 m}} F_{k}\left(x_{1}, \cdots, 2^{m} b_{k}, \cdots, x_{n}\right) \| \\
\leq \theta \cdot 2^{m(p-2)}\left(\left\|a_{k}\right\|^{p}+\left\|b_{k}\right\|^{p}\right)
\end{array}
$$

which tends to zero as $m \rightarrow \infty$. Thus we obtain

$$
\begin{aligned}
& \delta_{k}\left(x_{1}, \cdots, a_{k}+b_{k}, \cdots, x_{n}\right)+\delta_{k}\left(x_{1}, \cdots, a_{k}-b_{k}, \cdots, x_{n}\right) \\
& =2 \delta_{k}\left(x_{1}, \cdots, a_{k}, \cdots, x_{n}\right)+2 \delta_{k}\left(x_{1}, \cdots, b_{k}, \cdots, x_{n}\right)
\end{aligned}
$$

for all $a_{k}, b_{k} \in A_{k}$ and all $x_{i} \in A_{i}(i \neq k)$. Hence $\delta_{k}$ is quadratic with respect to the $k$-th variable. It follows from (2.7) that

$$
\left\|F_{k}\left(x_{1}, \cdots, x_{k}, \cdots, x_{n}\right)-\delta_{k}\left(x_{1}, \cdots, x_{k}, \cdots, x_{n}\right)\right\| \leq \frac{2 \theta}{4-2^{p}}\left\|x_{k}\right\|^{p}
$$

for all $x_{i} \in A_{i}(i=1,2, \cdots, n)$.

Replacing $a_{k}, b_{k}, c_{k}$ with $2^{m} a_{k}, 2^{m} b_{k}, 2^{m} c_{k}$, respectively, in (2.2), we obtain

$$
\begin{aligned}
& \| F_{k}\left(x_{1}, \cdots, 2^{3 m}\left[a_{k} b_{k} c_{k}\right], \cdots, x_{n}\right)- {\left[2^{2 m} g_{k}\left(a_{k}\right) 2^{2 m} g_{k}\left(b_{k}\right) F_{k}\left(x_{1}, \cdots, 2^{m} c_{k}, \cdots, x_{n}\right)\right] } \\
&- {\left[2^{2 m} g_{k}\left(a_{k}\right) F_{k}\left(x_{1}, \cdots, 2^{m} b_{k}, \cdots, x_{n}\right) 2^{2 m} g_{k}\left(c_{k}\right)\right] } \\
&- {\left[F_{k}\left(x_{1}, \cdots, 2^{m} a_{k}, \cdots, x_{n}\right) 2^{2 m} g_{k}\left(b_{k}\right) 2^{2 m} g_{k}\left(c_{k}\right)\right] \| } \\
& \leq 2^{m p} \cdot \theta\left(\left\|a_{k}\right\|^{p}+\left\|b_{k}\right\|^{p}+\left\|c_{k}\right\|^{p}\right) .
\end{aligned}
$$


Then we have

$$
\begin{array}{r}
\| \frac{1}{2^{6 m}} F_{k}\left(x_{1}, \cdots, 2^{3 m}\left[a_{k} b_{k} c_{k}\right], \cdots, x_{n}\right) \\
-\frac{1}{2^{6 m}}\left[2^{2 m} g_{k}\left(a_{k}\right) 2^{2 m} g_{k}\left(b_{k}\right) F_{k}\left(x_{1}, \cdots, 2^{m} c_{k}, \cdots, x_{n}\right)\right] \\
-\frac{1}{2^{6 m}}\left[2^{2 m} g_{k}\left(a_{k}\right) F_{k}\left(x_{1}, \cdots, 2^{m} b_{k}, \cdots, x_{n}\right) 2^{2 m} g_{k}\left(c_{k}\right)\right] \\
-\frac{1}{2^{6 m}}\left[F_{k}\left(x_{1}, \cdots, 2^{m} a_{k}, \cdots, x_{n}\right) 2^{2 m} g_{k}\left(b_{k}\right) 2^{2 m} g_{k}\left(c_{k}\right)\right] \| \\
\leq 2^{m(p-6)} \cdot \theta\left(\left\|a_{k}\right\|^{p}+\left\|b_{k}\right\|^{p}+\left\|c_{k}\right\|^{p}\right) .
\end{array}
$$

for all $a_{k}, b_{k}, c_{k} \in A_{k}$. Passing the limit $m \rightarrow \infty$ in above inequality, we obtain

$$
\begin{aligned}
\delta_{k}\left(x_{1}, \cdots,\left[a_{k} b_{k} c_{k}\right], \cdots, x_{n}\right) & =\left[g_{k}\left(a_{k}\right) g_{k}\left(b_{k}\right) \delta_{k}\left(x_{1}, \cdots, c_{k}, \cdots, x_{n}\right)\right] \\
& +\left[g_{k}\left(a_{k}\right) \delta_{k}\left(x_{1}, \cdots, b_{k}, \cdots, x_{n}\right) g_{k}\left(c_{k}\right)\right] \\
& +\left[\delta_{k}\left(x_{1}, \cdots, a_{k}, \cdots, x_{n}\right) g_{k}\left(b_{k}\right) g_{k}\left(c_{k}\right)\right]
\end{aligned}
$$

for all $a_{k}, b_{k}, c_{k} \in A_{k}$ and all $x_{i} \in A_{i}(i \neq k)$.

Finally, to prove the uniqueness of $\delta_{k}$, let $\delta_{k}^{\prime}: A_{1} \times \cdots \times A_{n} \rightarrow B$ be another $k$-th partial ternary quadratic derivation satisfying (2.3). Then we have

$$
\begin{aligned}
& \left\|\delta_{k}\left(x_{1}, \cdots, x_{n}\right)-\delta_{k}^{\prime}\left(x_{1}, \cdots, x_{n}\right)\right\| \\
& \quad=\frac{1}{2^{2 m}}\left\|\delta_{k}\left(x_{1}, \cdots, 2^{m} x_{k}, \cdots, x_{n}\right)-\delta_{k}^{\prime}\left(x_{1}, \cdots, 2^{m} x_{k}, \cdots, x_{n}\right)\right\| \\
& \quad \leq \frac{1}{2^{2 m}}\left(\left\|\delta_{k}\left(x_{1}, \cdots, 2^{m} x_{k}, \cdots, x_{n}\right)-F_{k}\left(x_{1}, \cdots, 2^{m} x_{k}, \cdots, x_{n}\right)\right\|\right. \\
& \left.\quad+\left\|F_{k}\left(x_{1}, \cdots, 2^{m} x_{k}, \cdots, x_{n}\right)-\delta_{k}^{\prime}\left(x_{1}, \cdots, 2^{m} x_{k}, \cdots, x_{n}\right)\right\|\right) \\
& \quad \leq \theta \sum_{i=m}^{\infty} 2^{i(p-2)}\left\|x_{k}\right\|^{p} .
\end{aligned}
$$

which tends to zero as $m \rightarrow \infty$ for all $x_{i} \in A_{i}(i=1,2, \cdots, n)$. So we can conclude that $\delta_{k}\left(x_{1}, \cdots, x_{n}\right)=\delta_{k}^{\prime}\left(x_{1}, \cdots, x_{n}\right)$. This proves the uniqueness of $\delta$.

Theorem 2.2. Let $p>2$ be and let $\theta$ be nonnegative real numbers. Let $F_{k}$ : $A_{1} \times \cdots \times A_{n} \rightarrow B$ be a mapping with $F_{k}\left(x_{1}, \cdots, 0_{k}, \cdots, x_{n}\right)=0_{B}$. Suppose that there exists a quadratic mapping $g_{k}: A_{k} \rightarrow B$ such that satisfying (2.1) and (2.2) for all $a_{k}, b_{k}, c_{k} \in A_{k}, x_{i} \in A_{i}(i \neq k)$. Then there exists a unique $k$-th partial ternary quadratic derivation $\delta_{k}: A_{1} \times \cdots \times A_{n} \rightarrow B$ such that

$$
\left\|F_{k}\left(x_{1}, \cdots, x_{n}\right)-\delta_{k}\left(x_{1}, \cdots, x_{n}\right)\right\| \leq \frac{2 \theta}{2^{p}-4}\left\|x_{k}\right\|^{p}
$$

holds for all $x_{i} \in A_{i}(i=1,2, \cdots, n)$.

Proof. In (2.1), putting $a_{k}=b_{k}=\frac{x_{k}}{2}$, we have

$$
\left\|F_{k}\left(x_{1}, \cdots, x_{k}, \cdots, x_{n}\right)-4 F_{k}\left(x_{1}, \cdots, \frac{x_{k}}{2}, \cdots, x_{n}\right)\right\| \leq \frac{2 \theta}{2^{p}}\left\|x_{k}\right\|^{p},
$$


for all $x_{i} \in A_{i}(i=1,2, \cdots, n)$. One can use induction on $m$ to show that

$$
\begin{gathered}
\left\|F_{k}\left(x_{1}, \cdots, x_{k}, \cdots, x_{n}\right)-2^{2 m} F_{k}\left(x_{1}, \cdots, \frac{x_{k}}{2^{m}}, \cdots, x_{n}\right)\right\| \\
\leq \frac{2 \theta}{2^{p}} \sum_{i=0}^{m-1} 2^{i(2-p)}\left\|x_{k}\right\|^{p}
\end{gathered}
$$

for all $x_{i} \in A_{i}(i=1,2, \cdots, n)$ and all non-negative integers $m$. Hence

$$
\begin{gathered}
\left\|2^{2 j} F_{k}\left(x_{1}, \cdots, \frac{x_{k}}{2^{j}}, \cdots, x_{n}\right)-2^{2(m+j)} F_{k}\left(x_{1}, \cdots, \frac{x_{k}}{2^{(m+j)}}, \cdots, x_{n}\right)\right\| \\
\leq \frac{2 \theta}{2^{p}} \sum_{i=j}^{m+j-1} 2^{i(2-p)}\left\|x_{k}\right\|^{p}
\end{gathered}
$$

for all non-negative integers $m$ and $j$ with $m \geq j$ and all $x_{i} \in A_{i}(i=1,2, \cdots, n)$. It follows from $p>2$ that the sequence $\left\{2^{2 m} F_{k}\left(x_{1}, \cdots, \frac{x_{k}}{2^{m}}, \cdots, x_{n}\right)\right\}$ is Cauchy. Due to the completeness of $B$, this sequence is convergent. So one can define the mapping $\delta_{k}: A_{1} \times \cdots \times A_{n} \rightarrow B$ given by

$$
\delta_{k}\left(x_{1}, \cdots, x_{n}\right)=\lim _{m \rightarrow \infty} 2^{2 m} F_{k}\left(x_{1}, \cdots, \frac{x_{k}}{2^{m}}, \cdots, x_{n}\right)
$$

for all $x_{i} \in A_{i}(i=1, \cdots, n)$. In $(2.1)$, replacing $a_{k}, b_{k}$ with $\frac{a_{k}}{2^{m}}, \frac{b_{k}}{2^{m}}$, respectively, we obtain that

$$
\begin{array}{r}
\| 2^{2 m} F_{k}\left(x_{1}, \cdots, \frac{a_{k}+b_{k}}{2^{m}}, \cdots, x_{n}\right)+2^{2 m} F_{k}\left(x_{1}, \cdots, \frac{a_{k}-b_{k}}{2^{m}}, \cdots, x_{n}\right) \\
-2 \cdot 2^{2 m} F_{k}\left(x_{1}, \cdots, \frac{a_{k}}{2^{m}}, \cdots, x_{n}\right)-2 \cdot 2^{2 m} F_{k}\left(x_{1}, \cdots, \frac{b_{k}}{2^{m}}, \cdots, x_{n}\right) \| \\
\leq \theta \cdot 2^{m(2-p)}\left(\left\|a_{k}\right\|^{p}+\left\|b_{k}\right\|^{p}\right)
\end{array}
$$

which tends to zero as $m \rightarrow \infty$. Thus we obtain

$$
\begin{array}{r}
\delta_{k}\left(x_{1}, \cdots, a_{k}+b_{k}, \cdots, x_{n}\right)+\delta_{k}\left(x_{1}, \cdots, a_{k}-b_{k}, \cdots, x_{n}\right) \\
=2 \delta_{k}\left(x_{1}, \cdots, a_{k}, \cdots, x_{n}\right)+2 \delta_{k}\left(x_{1}, \cdots, b_{k}, \cdots, x_{n}\right)
\end{array}
$$

for all $a_{k}, b_{k} \in A_{k}$ and all $x_{i} \in A_{i}(i \neq k)$. Hence $\delta_{k}$ is quadratic with respect to the $k$-th variable. It follows from (2.12) that

$$
\left\|F_{k}\left(x_{1}, \cdots, x_{k}, \cdots, x_{n}\right)-\delta_{k}\left(x_{1}, \cdots, x_{k}, \cdots, x_{n}\right)\right\| \leq \frac{2 \theta}{2^{p}-4}\left\|x_{k}\right\|^{p}
$$

for all $x_{i} \in A_{i}(i=1,2, \cdots, n)$.

Replacing $a_{k}, b_{k}, c_{k}$ with $\frac{a_{k}}{2^{m}}, \frac{b_{k}}{2^{m}}, \frac{c_{k}}{2^{m}}$, respectively, in (2.2), we obtain

$$
\begin{aligned}
\| F_{k}\left(x_{1}, \cdots, \frac{\left[a_{k} b_{k} c_{k}\right]}{2^{3 m}}, \cdots, x_{n}\right) & -\left[\frac{g_{k}\left(a_{k}\right)}{2^{2 m}} \frac{g_{k}\left(b_{k}\right)}{2^{2 m}} F_{k}\left(x_{1}, \cdots, \frac{c_{k}}{2^{m}}, \cdots, x_{n}\right)\right] \\
& -\left[\frac{g_{k}\left(a_{k}\right)}{2^{2 m}} F_{k}\left(x_{1}, \cdots, \frac{b_{k}}{2^{m}}, \cdots, x_{n}\right) \frac{g_{k}\left(c_{k}\right)}{2^{2 m}}\right] \\
& -\left[F_{k}\left(x_{1}, \cdots, \frac{a_{k}}{2^{m}}, \cdots, x_{n}\right) \frac{g_{k}\left(b_{k}\right)}{2^{2 m}} \frac{g_{k}\left(c_{k}\right)}{2^{2 m}}\right] \| \\
\leq & \frac{\theta}{2^{m p}}\left(\left\|a_{k}\right\|^{p}+\left\|b_{k}\right\|^{p}+\left\|c_{k}\right\|^{p}\right) .
\end{aligned}
$$


Then we have

$$
\begin{aligned}
\| 2^{6 m} F_{k}\left(x_{1}, \cdots, \frac{\left[a_{k} b_{k} c_{k}\right]}{2^{3 m}}, \cdots, x_{n}\right) & -2^{6 m}\left[\frac{g_{k}\left(a_{k}\right)}{2^{2 m}} \frac{g_{k}\left(b_{k}\right)}{2^{2 m}} F_{k}\left(x_{1}, \cdots, \frac{c_{k}}{2^{m}}, \cdots, x_{n}\right)\right] \\
& -2^{6 m}\left[\frac{g_{k}\left(a_{k}\right)}{2^{2 m}} F_{k}\left(x_{1}, \cdots, \frac{b_{k}}{2^{m}}, \cdots, x_{n}\right) \frac{g_{k}\left(c_{k}\right)}{2^{2 m}}\right] \\
& -2^{6 m}\left[F_{k}\left(x_{1}, \cdots, \frac{a_{k}}{2^{m}}, \cdots, x_{n}\right) \frac{g_{k}\left(b_{k}\right)}{2^{2 m}} \frac{g_{k}\left(c_{k}\right)}{2^{2 m}}\right] \| \\
& \leq 2^{m(6-p)} \cdot \theta\left(\left\|a_{k}\right\|^{p}+\left\|b_{k}\right\|^{p}+\left\|c_{k}\right\|^{p}\right) .
\end{aligned}
$$

for all $a_{k}, b_{k}, c_{k} \in A_{k}$. Passing the limit $m \rightarrow \infty$ in above inequality, we obtain

$$
\begin{aligned}
\delta_{k}\left(x_{1}, \cdots,\left[a_{k} b_{k} c_{k}\right], \cdots, x_{n}\right) & =\left[g_{k}\left(a_{k}\right) g_{k}\left(b_{k}\right) \delta_{k}\left(x_{1}, \cdots, c_{k}, \cdots, x_{n}\right)\right] \\
& +\left[g_{k}\left(a_{k}\right) \delta_{k}\left(x_{1}, \cdots, b_{k}, \cdots, x_{n}\right) g_{k}\left(c_{k}\right)\right] \\
& +\left[\delta_{k}\left(x_{1}, \cdots, a_{k}, \cdots, x_{n}\right) g_{k}\left(b_{k}\right) g_{k}\left(c_{k}\right)\right]
\end{aligned}
$$

for all $a_{k}, b_{k}, c_{k} \in A_{k}$ and all $x_{i} \in A_{i}(i \neq k)$.

Finally, to prove the uniqueness of $\delta_{k}$, let $\delta_{k}^{\prime}: A_{1} \times \cdots \times A_{n} \rightarrow B$ be another $k$-th partial ternary quadratic derivation satisfying (2.10). Then we have

$$
\begin{aligned}
\left\|\delta_{k}\left(x_{1}, \cdots, x_{n}\right)-\delta_{k}^{\prime}\left(x_{1}, \cdots, x_{n}\right)\right\| \\
\quad=2^{2 m}\left\|\delta_{k}\left(x_{1}, \cdots, \frac{x_{k}}{2^{m}}, \cdots, x_{n}\right)-\delta_{k}^{\prime}\left(x_{1}, \cdots, \frac{x_{k}}{2^{m}}, \cdots, x_{n}\right)\right\| \\
\quad \leq 2^{2 m}\left(\left\|\delta_{k}\left(x_{1}, \cdots, \frac{x_{k}}{2^{m}}, \cdots, x_{n}\right)-F_{k}\left(x_{1}, \cdots, \frac{x_{k}}{2^{m}}, \cdots, x_{n}\right)\right\|\right. \\
\left.\quad+\left\|F_{k}\left(x_{1}, \cdots, \frac{x_{k}}{2^{m}}, \cdots, x_{n}\right)-\delta_{k}^{\prime}\left(x_{1}, \cdots, \frac{x_{k}}{2^{m}}, \cdots, x_{n}\right)\right\|\right) \\
\quad \leq \theta \sum_{i=m}^{\infty} 2^{i(2-p)}\left\|x_{k}\right\|^{p} .
\end{aligned}
$$

which tends to zero as $m \rightarrow \infty$ for all $x_{i} \in A_{i}(i=1,2, \cdots, n)$. So we can conclude that $\delta_{k}\left(x_{1}, \cdots, x_{n}\right)=\delta_{k}^{\prime}\left(x_{1}, \cdots, x_{n}\right)$. This proves the uniqueness of $\delta$.

By Theorems 2.1 and 2.2 we solve the following Hyers-Ulam-Rassias stability problem.

Theorem 2.3. Let $\epsilon$ be nonnegative real numbers and let $F_{k}: A_{1} \times \cdots \times A_{n} \rightarrow B$ be a mapping with $F_{k}\left(x_{1}, \cdots, 0_{k}, \cdots, x_{n}\right)=0_{B}$. Assume that there exist a quadratic mapping $g_{k}: A_{k} \rightarrow B$ such that

$$
\begin{gathered}
\| F_{k}\left(x_{1}, \cdots, a_{k}+b_{k}, \cdots, x_{n}\right)+F_{k}\left(x_{1}, \cdots, a_{k}-b_{k}, \cdots, x_{n}\right) \\
-2 F_{k}\left(x_{1}, \cdots, a_{k}, \cdots, x_{n}\right)-2 F_{k}\left(x_{1}, \cdots, b_{k}, \cdots, x_{n}\right) \| \leq \epsilon \\
\| F_{k}\left(x_{1}, \cdots,\left[a_{k} b_{k} c_{k}\right], \cdots, x_{n}\right)-\left[g_{k}\left(a_{k}\right) g_{k}\left(b_{k}\right) F_{k}\left(x_{1}, \cdots, c_{k}, \cdots, x_{n}\right)\right] \\
-\left[g_{k}\left(a_{k}\right) F_{k}\left(x_{1}, \cdots, b_{k}, \cdots, x_{n}\right) g_{k}\left(c_{k}\right)\right] \\
-\left[F_{k}\left(x_{1}, \cdots, a_{k}, \cdots, x_{n}\right) g_{k}\left(b_{k}\right) g_{k}\left(c_{k}\right)\right] \| \leq \epsilon
\end{gathered}
$$


for all $a_{k}, b_{k}, c_{k} \in A_{k}, x_{i} \in A_{i}(i \neq k)$. Then there exists a unique $k$-th partial ternary quadratic derivation $\delta_{k}: A_{1} \times \cdots \times A_{n} \rightarrow B$ such that

$$
\left\|F_{k}\left(x_{1}, \cdots, x_{n}\right)-\delta_{k}\left(x_{1}, \cdots, x_{n}\right)\right\| \leq \frac{\epsilon}{3}
$$

holds for all $x_{i} \in A_{i}(i=1,2, \cdots, n)$.

Proof. In Theorem 2.1, by putting $p:=0$ and $\theta:=\frac{\epsilon}{2}$, we obtain the conclusion of the theorem.

\section{REFERENCES}

[1] S. Abbaszadeh, Intuitionistic fuzzy stability of a quadratic and quartic functional equation, Int. J. Nonlinear Anal. Appl. 1 (2010),2, 100-124.

[2] R. Badora, On approximate derivations, Math. Inequal. Appl. 9 (2006), 167-173.

[3] M. Bavand Savadkouhi, M. Eshaghi Gordji, J. M. Rassias and N. Ghobadipour, Approximate ternary Jordan derivations on Banach ternary algebras, J. Math. Phys, 50 (2009), 9 pages.

[4] P. W. Cholewa, Remarks on the stability of functional equations, Aequationes Math. 27 (1984), 76-86.

[5] H. Chu, S. Koo and J. Park, Partial stabilities and partial derivations of $n$-variable functions, Nonlinear Anal.-TMA (to appear).

[6] S. Czerwik, On the stability of the quadratic mapping in normed spaces, Abh. Math. Sem. Univ. Hamburg 62 (1992), 59-64.

[7] A. Ebadian, A. Najati, M. E. Gordji, On approximate additive-quartic and quadraticcubic functional equations in two variables on abelian groups, Results Math. DOI 10.1007/s00025-010-0018-4 (2010).

[8] M. Eshaghi Gordji, Stability of a functional equation deriving from quartic and additive functions, Bull. Korean Math. Soc. Vol. 47, No.3, (2010), 491-502.

[9] M. Eshaghi Gordji, Stability of an additive-quadratic functional equation of two variables in F-spaces, Journal of Nonlinear Sciences and Applications, Vol 2, No 4,(2009) pp. 251-259.

[10] M. Eshaghi Gordji, S. Abbaszadeh and C. Park, On the stability of generalized mixed type quadratic and quartic functional equation in quasi-Banach spaces, J. Ineq. Appl. (2009), Article ID 153084, 26 pages.

[11] M. Eshaghi Gordji, M. Bavand Savadkouhi and C. Park, Quadratic-quartic functional equations in RN-spaces, J. Ineq. Appl. (2009), Article ID 868423, 14 pages.

[12] M. Eshaghi Gordji, M. Bavand Savadkouhi and M. Bidkham, Stability of a mixed type additive and quadratic functional equation in non-Archimedean spaces, Journal of Computational Analysis and Applications. VOL. 12, No. 2, (2010), 454-462.

[13] M. Eshaghi Gordji and A. Bodaghi, On the Hyers-Ulam-Rasias Stability problem for quadratic functional equations, East Journal On Approximations. Vol. 16, No. 2 (2010), $123-130$.

[14] M. Eshaghi Gordji and A. Bodaghi, On the stability of quadratic double centralizers on Banach algebras, J. Comput. Anal. Appl. (in press).

[15] M. Eshaghi Gordji, M. B. Ghaemi, S. Kaboli Gharetapeh, S. Shams, A. Ebadian, On the stability of $J^{*}$-derivations, Journal of Geometry and Physics. 60(3) (2010), 454-459.

[16] M. Eshaghi Gordji, M. Ghanifard, H. Khodaei and C. Park, A fixed point approach to the random stability of a functional equation driving from quartic and quadratic mappings, Discrete Dynamics in Nature and Society. (2010), Article ID: 670542.

[17] M. Eshaghi Gordji, N. Ghobadipour, Stability of $(\alpha, \beta, \gamma)$-derivations on Lie $C^{*}$-algebras, To appear in International Journal of Geometric Methods in Modern Physics (IJGMMP). 
[18] M. Eshaghi Gordji, S. Kaboli Gharetapeh, T. Karimi , E. Rashidi and M. Aghaei, Ternary Jordan derivations on $C^{*}$-ternary algebras, Journal of Computational Analysis and Applications, VOL.12, No.2, 2010, 463-470.

[19] M. Eshaghi Gordji, S. Kaboli-Gharetapeh, C. Park and S. Zolfaghri, Stability of an additive-cubic-quartic functional equation, Advances in Difference Equations. (2009), Article ID 395693, 20 pages.

[20] M. Eshaghi Gordji, S. Kaboli Gharetapeh, J. M. Rassias and S. Zolfaghari, Solution and stability of a mixed type additive, quadratic and cubic functional equation, Advances in difference equations. Volume 2009, Article ID 826130, 17 pages, doi:10.1155/2009/826130.

[21] M. Eshaghi Gordji, H. Khodaei, On the Generalized Hyers-Ulam-Rassias Stability of Quadratic Functional Equations, Abs. Appl. Anal. Volume 2009, Article ID 923476, 11 pages.

[22] M. Eshaghi Gordji, H. Khodaei, Solution and stability of generalized mixed type cubic, quadratic and additive functional equation in quasi-Banach spaces, Nonlinear AnalysisTMA 71 (2009), 5629-5643.

[23] M. Eshaghi Gordji and M. S. Moslehian, A trick for investigation of approximate derivations, Math. Commun. 15 (2010), no. 1, 99-105.

[24] M. Eshaghi Gordji, M. Ramezani, A. Ebadian and C. Park, Quadratic double centralizers and quadratic multipliers, Advances in Difference Equations (in press).

[25] M. Eshaghi Gordji, J. M. Rassias, N. Ghobadipour, Generalized Hyers-Ulam stability of the generalized $(n, k)$-derivations, Abs. Appl. Anal., Volume 2009, Article ID 437931, 8 pages.

[26] R. Farokhzad and S. A. R. Hosseinioun, Perturbations of Jordan higher derivations in Banach ternary algebras: An alternative fixed point approach, Internat. J. Nonlinear Anal. Appl. 1 (2010), 1, 42-53.

[27] Z. Gajda, On stability of additive mappings, Internat. J. Math. Sci. 14 (1991), 431-434.

[28] P. Găvruta, A generalization of the Hyers-Ulam-Rassias stability of approximately additive mappings, J. Math. Anal. Appl, 184 (1994), 431-436.

[29] A. Grabiec, The generalized Hyers-Ulam stability of a class of functional equations, Publ. Math. Debrecen. 48 (1996), 217-235.

[30] D. H. Hyers, G. Isac and Th. M. Rassias, Stability of functional equations in several variables, Birkhaĕr, Basel, (1998).

[31] D. H. Hyers, On the stability of the linear functional equation, Proc. Natl. Acad. Sci, 27 (1941), 222-224.

[32] G. Isac and Th. M. Rassias, On the Hyers-Ulam stability of $\psi$-additive mappings, J. Approx. Theory, 72 (1993), 131-137.

[33] K. Jun and D. Park, Almost derivations on the Banach algebra $C^{n}[0,1]$, Bull. Korean Math. Soc. 33 (1996), 359-366.

[34] Pl. Kannappan, Quadratic functional equation and inner product spaces, Results Math, 27 (1995), 368-372.

[35] H. Khodaei and M. Kamyar, Fuzzy approximately additive mappings, Int. J. Nonlinear Anal. Appl. 1 (2010), 2, 44-53.

[36] H. Khodaei and Th. M. Rassias, Approximately generalized additive functions in several variables, Int. J. Nonlinear Anal. Appl. 1 (2010),1, 22-41.

[37] C. Park and M. Eshaghi Gordji, Comment on Approximate ternary Jordan derivations on Banach ternary algebras [Bavand Savadkouhi et al. J. Math. Phys. 50, 042303 (2009)], J. Math. Phys. 51, 044102 (2010) (7 pages).

[38] C. Park and A. Najati, Generalized additive functional inequalities in Banach algebras, Int. J. Nonlinear Anal. Appl. 1 (2010),2, 54-62.

[39] Th. M. Rassias, On the stability of the linear mapping in Banach spaces, Proc. Amer. Math. Soc. 72 (1978), 297-300.

[40] F. Skof, Propriet locali e approssimazione di operatori, Rend. Sem. Mat. Fis. Milano, 53 (1983), 113-129. 
[41] S. M. Ulam, Problems in modern mathematics, Chapter VI, science ed., Wiley, New York, (1940).

${ }^{1}$ Department of Physics, Semnan University, P. O. Box 35195-363, Semnan, Iran

${ }^{2,3}$ Department of Mathematics, Semnan University, P. O. Box 35195-363, SemNAN, IRAN

E-mail address: madjid.eshaghi@gmail.com; bavand.m@gmail.com 